\title{
Labral tears with axial plane disorders
}

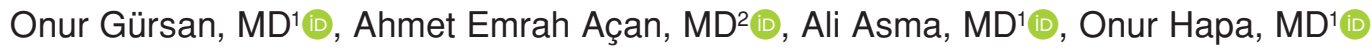 \\ ${ }^{1}$ Department of Orthopedics and Traumatology, Faculty of Medicine Dokuz Eylül University, Izmir, Turkey \\ ${ }^{2}$ Department of Orthopedics and Traumatology, Faculty of Medicine Muğla Sıtkı Koçman University, Muğla, Turkey
}

Femoroacetabular impingement (FAI) leads to a disruption of the chondrolabral junction and/or labral tears with two main mechanisms; cam type impingement on the aspherical femoral head-neck junction abutting against the acetabulum or pincer type with excessive acetabular coverage, leading to labral tears or fraying. Both may be a common reason for early-onset hip osteoarthritis. ${ }^{[1-3]}$ However, such anatomical findings are also reported to be relevant among asymptomatic people, or the contralateral asymptomatic side might have similar findings on radiographs compared to the symptomatic side in patients with the impingement. ${ }^{[4-6]}$

Recently, it was reported that patients with acetabular retroversion had developed hip pain earlier than the patients with acetabular anteversion (AA) regardless of the severity of the dysplasia. Acetabular retroverted hips had normal anterior axial plane coverage as well as deficient posterior coverage. Authors argued against the traditional belief that the development of secondary osteoarthritis due to labral tears or cartilage degeneration appears to be less affected by posterior dysplasia than anterior dysplasia and that posterior acetabular coverage particularly seems to play a role in the development of osteoarthritis. ${ }^{[7,8]}$ Supporting this, Hapa et al. ${ }^{[9]}$ reported axial plane posterior

Received: June 25, 2019

Accepted: December 11, 2019

Published online: March 02, 2020

Correspondence: Onur Gürsan, MD. Dokuz Eylül Üniversitesi Tıp Fakültesi Ortopedi ve Travmatoloji Anabilim Dalı, 35340 İnciraltı, İzmir, Türkiye.

E-mail: onur_84_gursan@hotmail.com

Doi: $10.5606 /$ ehc. 2020.70193

Citation: Gürsan O, Açan AE, Asma A, Hapa O. Labral tears with axial plane disorders. Jt Dis Relat Surg 2020;31(1):109-114.

\section{ABSTRACT}

Objectives: This study aims to determine if there is an axial plane coverage insufficiency in patients with symptomatic labral tears compared to the contralateral asymptomatic side and healthy control subjects.

Patients and methods: This retrospective study was conducted between December 2017 and January 2019. Thirty patients (21 males, 9 females; mean age 28 years; range, 20 to 36 years) operated due to unilateral symptomatic acetabular labral tears secondary to femoroacetabular impingement were evaluated. Twenty asymptomatic patients ( 13 males, 7 females; mean age $27 \pm 9$ years; range, 19 to 36 years) were included in the control group. The relationship between acetabular morphology and labral tear was investigated with the comparison of unilateral symptomatic hips with contralateral asymptomatic hips and the control group by using radiological parameters on plain radiographs and computed tomography.

Results: When the patient group symptomatic side was compared to the control group, acetabular anteversion angle (AAA) and alpha $(\alpha)$ angle were higher, while posterior acetabular sector angle and horizontal acetabular sector angle were lower. When the asymptomatic side was compared to the control group, AAA was higher in the patient group. There was no difference between the symptomatic and asymptomatic sides in the patient group; the symptomatic side yielded a higher $\alpha$ angle.

Conclusion: Posterior axial plane coverage deficiency in combination with cam deformity (increased $\alpha$ angle) seems to play a role in the pathogenesis of symptomatic acetabular labral tears, even creating a side-to-side difference in some individuals.

Keywords: Acetabular anteversion angle, alpha angle, axial plane coverage, femoroacetabular impingement, hip dysplasia, labral tear.

acetabular coverage deficiency in patients with hip osteoarthritis. Hingsammer et al. ${ }^{[10]}$ reported that acetabular coverage increases with skeletal maturity as a result of the growth of the posterior wall and that acetabular retroversion seems to be due to insufficient growth of the posterior wall rather than an overgrowth of the anterior wall.

Based on the above-mentioned studies, we hypothesized that patients with symptomatic labral 
tears would have axial plane posterior coverage insufficiency. Therefore, in this study, we aimed to determine if there is an axial plane coverage deficiency in patients with symptomatic labral tears compared to the contralateral asymptomatic side and healthy control subjects.

\section{PATIENTS AND METHODS}

This retrospective study was conducted at Dokuz Eylül University Faculty of Medicine Hospital between December 2017 and January 2019. Thirty patients (21 males, 9 females; mean age 28 years; range, 20 to 36 years) operated due to unilateral symptomatic acetabular labral tears secondary to FAI were evaluated. Unilateral hip pain was the main symptom, and anterior impingement tests were positive in this group. The labral tear was confirmed by both preoperative imaging studies (magnetic resonance imaging) and intraoperatively during hip arthroscopy. Labral repair and femoroplasty were performed. Patients having Legg-CalvePerthes disease, slipped capital femoral epiphysis, congenital dislocation, infection, rheumatoid or other inflammatory diseases, trauma, previous hip surgery, or hip osteoarthritis or dysplasia were excluded. Sixteen of the included patients had a left-sided disease, while 14 had a right-sided disease.

The control group constituted gender- and age-matched 20 asymptomatic patients (13 males, 7 females; mean age $27 \pm 9$ years; range, 19 to 36 years) for the hips and without any known connective tissue disorder, examined by radiography and computed tomography (CT) of the abdominal and/or pelvic area due to the symptoms of the abdominal area or with a suspected disease in the pelvic or abdominal region. The study protocol was approved by the Dokuz Eylül University Faculty of Medicine Ethics Committee. A written informed consent was obtained from each participant. The study was conducted in accordance with the principles of the Declaration of Helsinki.

The CT device was the same in all patients, including the control group. The median radiation dose was also similar (6 mSv) between the two groups. Patients were scanned according to standard departmental protocols at $120 \mathrm{kVp}$ and 140 to $180 \mathrm{mAs}$ depending on patient weight and/or girth. Patients were positioned supine and neutral rotation in both groups within the framework of standard departmental protocols. Axial CT images with $3 \mathrm{~mm}$ slice thickness were obtained in both groups with a GE Prospeed SX CT system (General Electric Medical Systems, Milwaukee, WI, USA). Pelvic tilt and rotation affect the radiographic analysis of the hip joint. ${ }^{[11-13]}$ To eliminate this issue, we reformatted the CT images into standardized pelvic images with neutral tilt by using Sectra Workstation IDS7 V20.2.10.3376 (Sectra AB, Linköping, Sweden). Three-dimensional $\mathrm{CT}$ scans were used for reformatting by aligning the left and right superior iliac spines in axial view, superior portion of the right and the left iliac spines in the coronal plane, and by aligning the pubic symphysis and the anterior superior iliac spine in the sagittal plane. After reorientation, the CT scan was reformatted and the radiologic assessment was performed. ${ }^{[14]}$

The center of edge angle (CEA) on the pelvis radiographs and CT scenographs (control group), alpha $(\alpha)$ angle, acetabular anteversion angle (AAA), anterior acetabular sector angle (AASA), posterior acetabular sector angle (PASA), and horizontal acetabular sector angle (HASA) were measured by $\mathrm{CT}$ examinations. The $\alpha$ angle was calculated via obtaining an angled axial plane parallel to the axis of the femoral neck and passing through the center of the femoral head. The femoral neck axis line was defined in this image. The best matching circumference was drawn over the femoral head contour. The angle was then calculated between the head-neck axis and the point where the femoral head outlines the circumference anteriorly. ${ }^{[15]}$

While measuring the AA, the obliquity caused by improper positioning of the patient in the CT scanner was controlled by drawing a baseline intersecting the most posterior edges of the ilium. Acetabular anteversion angle describes the angulation of a line

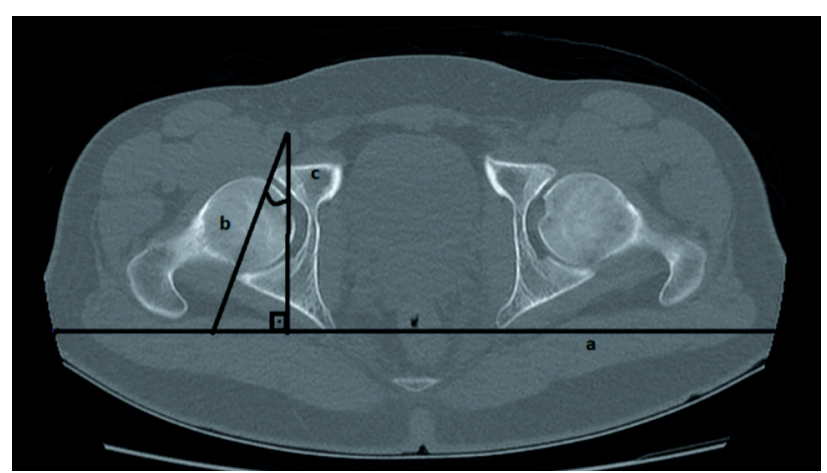

FIGURE 1. Measurement of acetabular anteversion on computed tomography. Acetabular anteversion angle is measured as angle between a line between anterior and posterior acetabular ridge (line b) and a reference line (line c) drawn perpendicular to a line between posterior pelvic margins at level of sciatic notch (line a). 


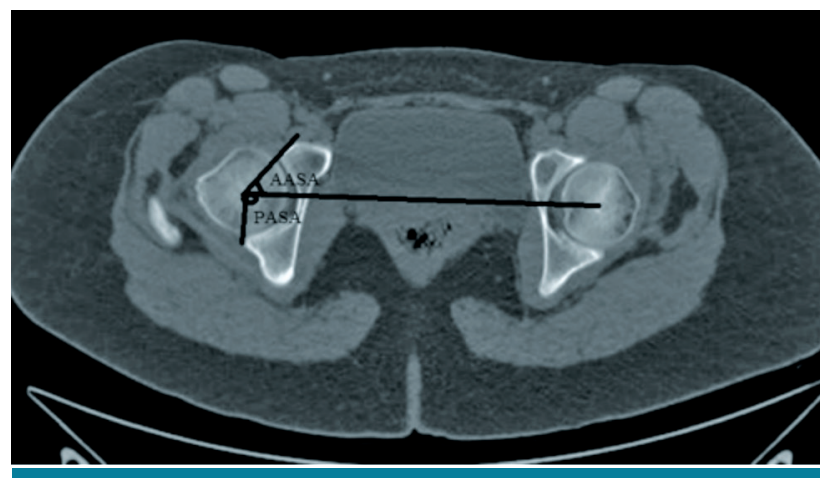

FIGURE 2. Measurement of anterior acetabular sector angle (angle between anterior acetabular margin, center of femoral head, and intercapital center line) and posterior acetabular sector angle (angle between posterior acetabular margin, center of femoral head, and intercapital center line).

through the anterior lip of the acetabulum and the lip of the posterior acetabulum with the sagittal plane (Figure 1) ${ }^{[16]}$ The AASA and PASA were measured in the equatorial plane in the axial plane sections, just as Anda et al. had performed. ${ }^{[17,18]}$ The equatorial plane was defined as the plane passing axially through the centers of the femoral heads. We used concentric circles for detecting the center of the femoral heads. Anterior acetabular sector angle was defined as the angle between the anterior acetabular margin, the center of the femoral head, and the intercapital centerline. Posterior acetabular sector angle was described as the angle between the posterior acetabular margin, the center of the femoral head, and the intercapital centerline (Figure 2). Horizontal acetabular sector angle was defined as the total axial plane coverage angle calculated as the sum of AASA and PASA (AASA+PASA). The measurements were obtained from CT images by two independent observers blinded to the patients and the scope of the study.

\section{Statistical analysis}

The SPSS for Windows version 10.1 (SPSS Inc., Chicago, IL, USA) was used to analyze the data. A t-test was used to compare groups regarding age and a chi-square test regarding gender. A paired samples test was used to compare the symptomatic side to the contralateral asymptomatic side of the patients. A covariance analysis test was used to compare the patient group with the control group. We evaluated intra- and interobserver variabilities using kappa coefficients with Fisher's exact test. For the intraobserver variability (based on two measurements separated by a three-week interval), the kappa was 0.83 . For interobserver variability, the kappa was 0.76 .

\section{RESULTS}

Demographic data are given in Table I. There was no difference between the patient and control groups regarding age and gender. The patient and control groups' data are given in Table II. There was no significant difference between the symptomatic and asymptomatic contralateral sides in the patient group, whereas the $\alpha$ angle tended to be higher on the symptomatic side $(\mathrm{p}=0.053)$.

When the patient group symptomatic side was compared to the control group, AA and $\alpha$ angle were higher ( $\mathrm{p}=0.02, \mathrm{p}=0.02)$, while PASA and HASA were lower ( $p=0.04, p=0.02$ ). When the asymptomatic side was compared to the control group, AA was higher in the patient group $(\mathrm{p}=0.03)$.

\section{DISCUSSION}

The principal finding of this study was that patients with labral tears showed posterior coverage deficiency and higher axial $\alpha$ angle on CT sections, compared to the control subjects, whereas this was not observed in the contralateral asymptomatic side. ${ }^{[19]}$ Side-to-side comparison of the patient group yielded no difference

\begin{tabular}{|c|c|c|c|c|c|c|c|}
\hline \multicolumn{8}{|c|}{$\begin{array}{l}\text { TABLE I } \\
\text { Demographic data for patient and control groups }\end{array}$} \\
\hline & \multicolumn{3}{|c|}{ Patient group } & \multicolumn{3}{|c|}{ Control group } & \multirow[b]{2}{*}{$p$} \\
\hline & $\mathrm{n}$ & $\%$ & Mean $\pm S D$ & $n$ & $\%$ & Mean $\pm S D$ & \\
\hline Age (year) & & & $28 \pm 8$ & & & $27 \pm 9$ & 0.425 \\
\hline \multicolumn{8}{|l|}{ Gender } \\
\hline Male & 21 & 70 & & 13 & 65 & & 0.425 \\
\hline \multicolumn{8}{|c|}{ Affected side } \\
\hline Left & 16 & & & - & & & \\
\hline Right & 14 & & & - & & & \\
\hline
\end{tabular}




\begin{tabular}{|c|c|c|c|c|c|c|}
\hline \multicolumn{7}{|c|}{$\begin{array}{c}\text { TABLE II } \\
\text { Measured parameters }\end{array}$} \\
\hline & $\mathrm{CE}\left({ }^{\circ}\right)$ & Alpha $\left({ }^{\circ}\right)$ & $\mathrm{AA}\left({ }^{\circ}\right)$ & AASA $\left(^{\circ}\right)$ & PASA $\left({ }^{\circ}\right)$ & $\operatorname{HASA}\left({ }^{\circ}\right)$ \\
\hline & Mean $\pm S D$ & Mean $\pm S D$ & Mean $\pm S D$ & Mean $\pm S D$ & Mean $\pm S D$ & Mean $\pm S D$ \\
\hline \multicolumn{7}{|l|}{ Patient group } \\
\hline Normal side & $38 \pm 6$ & $56 \pm 12$ & $17 \pm 7$ & $59 \pm 10$ & $94 \pm 9$ & $153 \pm 16$ \\
\hline Affected side & $41 \pm 8$ & $58 \pm 14$ & $19 \pm 8$ & $58 \pm 8$ & $91 \pm 11$ & $149 \pm 14$ \\
\hline Control group & $40 \pm 5$ & $48 \pm 7$ & $16 \pm 2$ & $64 \pm 9$ & $101 \pm 8$ & $165 \pm 15$ \\
\hline
\end{tabular}

except that the symptomatic side's $\alpha$ angle tended to be higher $(\mathrm{p}=0.054)$.

Recently, Hingsammer et al. ${ }^{[10]}$ reported that acetabular version and acetabular sector angles increase with the growth of the posterior wall. Based on this, they proposed that pincer-type impingement may not be due to overgrowth of the anterior wall but rather it may be due to insufficient growth of the posterior wall. Supporting this, Hapa et al. ${ }^{[9]}$ reported axial posterior coverage insufficiency in patients with primary hip osteoarthritis where sphericity of the femoral head is conserved. The AASA and PASA values were similar to those of the present study (AASA: $67^{\circ}$, PASA: $104^{\circ}$ ). Also, similar to the present study, they reported lower PASA in osteoarthritic hips (PASA: $96^{\circ}$ ), while no difference was noted for a normal asymptomatic contralateral side (PASA: 104 ${ }^{\circ}$ ) compared to the control group. They did not report any difference regarding total coverage (HASA), unlike the present study. This may be explained by the fact that although statistically insignificant, the AASA values were lower in the patient group, and this was contributing to the lower HASA values that were observed.

Acetabular anteversion angle was interestingly found to be higher in the patient group compared to the control group, while similar to that reported in the literature $\left(19^{\circ} \pm 4.5^{\circ}\right.$ and $21^{\circ} \pm 5^{\circ}$, respectively). ${ }^{[20,21]}$ Fujii et al.$^{[8]}$ have speculated that posterior dysplasia mainly plays a role in the pathogenesis of labral tears, chondral damage, and secondary osteoarthritis development. Furthermore, they have reported that the onset of pain was seen at an earlier age in patients with acetabular retroversion than hips with anterosuperior dysplasia. Within the presence of high contact pressures at the posterosuperior parts of the acetabulum during daily activities ${ }^{[22]}$ and considering the younger population in their study, posterior insufficiency might lead to increased stress concentration. Likewise, intraarticular pathologies such as labral tears may occur due to increased contact pressure with anterosuperior insufficiency. ${ }^{[23]}$ At this point, we believe that studies indicating the localization of intraarticular pathologies as well as labral tears will contribute more significantly in terms of acetabular insufficiency.

In contrast to the present study and the studies mentioned above, Valera et al. ${ }^{[24]}$ reported higher AASA, PASA, and HASA values in patients with grade 1 or 2 osteoarthritis and also found that higher HASA is correlated with higher CEA $\left(>35^{\circ}\right)$. They explained that this difference might be attributable to the inclusion of only a younger patient group with early-stage osteoarthritis in comparison with the mean age in the study of Hapa et al. ${ }^{[9]}$ Normal values of AASA and PASA have been described as approximately $63^{\circ}$ and $103^{\circ}$, respectively, ${ }^{[17,24,25]}$ and anterior dysplasia was defined when AASA was $<50^{\circ}$ and posterior dysplasia PASA was $<90^{\circ} .{ }^{[17]}$ No subject in the patient group of our study had obvious dysplasia according to these criteria (AASA, PASA, and HASA being $63^{\circ}, 100^{\circ}$, and $162^{\circ}$, respectively). Although the present study had a smaller sample size, it could be noted that both the patient and control groups were younger than the population of their study. So, the age of patients cannot be the only parameter about this discrepancy. Moreover, they did not consider pelvic tilt while measuring hips. The effects of pelvic tilt on hip radiological parameters might explain these differences in AASA, PASA, and HASA.

In their experimental and clinical study, Tannast et al. ${ }^{[13]}$ confirmed that lateral CEA, craniocaudal acetabular coverage, and depth of acetabulum showed no alteration with any changes in pelvic orientation and also explained that these parameters are independent of individual pelvic tilt and rotation in clinical practice. Anteroposterior acetabular coverage, crossover, and posterior wall sign, and retroversion index need specific efforts to determine pelvis orientation such 
as computer-assisted evaluation of radiographs. Although we reformatted and reoriented pelvis in a neutral position, a standardized method that includes pelvic tilt and rotation should be enhanced in terms of optimal patient positioning in the course of getting CT images such as an inclinometer applied to pelvis or ultrasonography-assisted techniques. ${ }^{[26,27]}$

This study has some limitations. The first limitation is the data being collected retrospectively. Secondly, the sample size was smaller than compared to the literature. However, despite the smaller sample size, most of the non-significant results, such as the $\alpha$ angle, were congruent with previous reports. The third limitation is the degree of the labral tears not being graded. It may be more valuable if labral tear localization and degree of tears were noted in terms of high contact pressures theory and acetabular version effects. The presence of a retroverted femur seems to be a cofactor in the development of hip osteoarthritis in patients with FAI. ${ }^{[28]}$ Another limitation is the absence of information about the femoral version values of the patients.

In conclusion, posterior axial plane coverage deficiency, in combination with cam deformity (increased $\alpha$ angle), seems to play a role in the pathogenesis of symptomatic hip labral tears and may even create a side-to-side difference in an individual. We recommend keeping horizontal plane acetabular disorders in mind and including parameters such as AASA, PASA, and HASA while evaluating patients in terms of hip preservation surgery.

\section{Declaration of conflicting interests}

The authors declared no conflicts of interest with respect to the authorship and/or publication of this article.

\section{Funding}

The authors received no financial support for the research and/or authorship of this article.

\section{REFERENCES}

1. Beck M, Kalhor M, Leunig M, Ganz R. Hip morphology influences the pattern of damage to the acetabular cartilage: femoroacetabular impingement as a cause of early osteoarthritis of the hip. J Bone Joint Surg [Br] 2005;87:1012-8.

2. Polat G, Şahin K, Arzu U, Kendirci AŞ, Aşık M. Prevalence of asymptomatic femoroacetabular impingement in Turkey; cross sectional study. Acta Orthop Traumatol Turc 2018;52:49-53.

3. Aydin M, Kircil C, Polat O, Arikan M, Erdemli B. Adult acetabulo - pelvic parameters in Turkish society: A descriptive radiological study. Acta Orthop Traumatol Turc 2016;50:623-7.

4. Tanzer M, Noiseux N. Osseous abnormalities and early osteoarthritis: the role of hip impingement. Clin Orthop Relat Res 2004;429:170-7.
5. Hack K, Di Primio G, Rakhra K, Beaulé PE. Prevalence of cam-type femoroacetabular impingement morphology in asymptomatic volunteers. J Bone Joint Surg [Am] 2010;92:2436-44

6. Larson CM, Moreau-Gaudry A, Kelly BT, Byrd JW, Tonetti J, Lavallee S, et al. Are normal hips being labeled as pathologic? A CT-based method for defining normal acetabular coverage. Clin Orthop Relat Res 2015;473:1247-54.

7. Nardo L, Parimi N, Liu F, Lee S, Jungmann PM, Nevitt MC, et al. Femoroacetabular impingement: Prevalent and often asymptomatic in older men: The osteoporotic fractures in men study. Clin Orthop Relat Res 2015;473:2578-86.

8. Fujii M, Nakashima Y, Yamamoto T, Mawatari T, Motomura G, Matsushita A, et al. Acetabular retroversion in developmental dysplasia of the hip. J Bone Joint Surg [Am] 2010;92:895-903.

9. Hapa O, Yüksel HY, Muratlı HH, Akşahin E, Gülçek S, Celebi $\mathrm{L}$, et al. Axial plane coverage and torsion measurements in primary osteoarthritis of the hip with good frontal plane coverage and spherical femoral head. Arch Orthop Trauma Surg 2010;130:1305-10.

10. Hingsammer AM, Bixby S, Zurakowski D, Yen YM, Kim YJ. How do acetabular version and femoral head coverage change with skeletal maturity? Clin Orthop Relat Res 2015;473:1224-33.

11. Siebenrock KA, Kalbermatten DF, Ganz R. Effect of pelvic tilt on acetabular retroversion: a study of pelves from cadavers. Clin Orthop Relat Res 2003;407:241-8.

12. Dandachli W, Islam SU, Liu M, Richards R, Hall-Craggs M, Witt J. Three-dimensional CT analysis to determine acetabular retroversion and the implications for the management of femoro-acetabular impingement. J Bone Joint Surg [Br] 2009;91:1031-6.

13. Tannast M, Fritsch S, Zheng G, Siebenrock KA, Steppacher $\mathrm{SD}$. Which radiographic hip parameters do not have to be corrected for pelvic rotation and tilt? Clin Orthop Relat Res 2015;473:1255-66.

14. Monazzam S, Bomar JD, Dwek JR, Hosalkar HS, Pennock AT. Development and prevalence of femoroacetabular impingement-associated morphology in a paediatric and adolescent population: a CT study of 225 patients. Bone Joint J 2013;95-B:598-604.

15. Nötzli HP, Wyss TF, Stoecklin CH, Schmid MR, Treiber K, Hodler J. The contour of the femoral head-neck junction as a predictor for the risk of anterior impingement. J Bone Joint Surg [Br] 2002;84:556-60.

16. Weiner LS, Kelley MA, Ulin RI, Wallach D. Development of the acetabulum and hip: computed tomography analysis of the axial plane. J Pediatr Orthop 1993;13:421-5.

17. Anda S, Svenningsen S, Dale LG, Benum P. The acetabular sector angle of the adult hip determined by computed tomography. Acta Radiol Diagn (Stockh) 1986;27:443-7.

18. Anda S, Terjesen T, Kvistad KA, Svenningsen S. Acetabular angles and femoral anteversion in dysplastic hips in adults: CT investigation. J Comput Assist Tomogr 1991;15:115-20.

19. Atik OŞ. Is there something new and interesting in my article? Eklem Hastalik Cerrahisi 2019;30:69.

20. Akiyama M, Nakashima Y, Fujii M, Sato T, Yamamoto T, Mawatari T, et al. Femoral anteversion is correlated with acetabular version and coverage in Asian women with 
anterior and global deficient subgroups of hip dysplasia: a CT study. Skeletal Radiol 2012;41:1411-8.

21. Ito $H$, Matsuno $T$, Hirayama $T$, Tanino $H$, Yamanaka Y, Minami A. Three-dimensional computed tomography analysis of non-osteoarthritic adult acetabular dysplasia. Skeletal Radiol 2009;3:131-9.

22. Witte H, Eckstein F, Recknagel S. A calculation of the forces acting on the human acetabulum during walking. Based On in vivo force measurements, kinematic analysis and morphometry. Acta Anat (Basel) 1997;160:269-80.

23. McCarthy JC, Lee JA. Acetabular dysplasia: a paradigm of arthroscopic examination of chondral injuries. Clin Orthop Relat Res 2002;405:122-8.

24. Valera M, Ibáñez N, Sancho R, Llauger J, Gich I. Acetabular overcoverage in the horizontal plane: an underdiagnosed trigger of early hip arthritis. A CT scan study in young adults. Arch Orthop Trauma Surg 2018;138:73-82.

25. Dolan MM, Heyworth BE, Bedi A, Duke G, Kelly BT. CT reveals a high incidence of osseous abnormalities in hips with labral tears. Clin Orthop Relat Res 2011;469:831-8.

26. Anda S, Svenningsen S, Grontvedt T, Benum P. Pelvic inclination and spatial orientation of the acetabulum. A radiographic, computed tomographic and clinical investigation. Acta Radiol 1990;31:389-94.

27. Lembeck B, Mueller O, Reize P, Wuelker N. Pelvic tilt makes acetabular cup navigation inaccurate. Acta Orthop 2005;76:517-23.

28. Zeng WN, Wang FY, Chen C, Zhang Y, Gong XY, Zhou K, et al. Investigation of association between hip morphology and prevalence of osteoarthritis. Sci Rep 2016;6:23477. 